\title{
Lymph node micrometastases detected by carcinoembryonic antigen mRNA affect long-term survival and disease-free interval in early-stage lung cancer patients
}

\author{
MARIO NOSOTTI ${ }^{1}$, ALESSANDRO PALLESCHI ${ }^{1}$, LORENZO ROSSO $^{1}$, DAVIDE TOSI $^{1}$, LUIGI SANTAMBROGIO ${ }^{1}$, \\ PAOLO MENDOGNI ${ }^{1}$, ANTONELLA MARZORATI ${ }^{2}$, ILARIA RIGHI $^{1}$ and SILVANO BOSARI ${ }^{2}$ \\ ${ }^{1}$ Thoracic Surgery and Lung Transplantation Unit; ${ }^{2}$ Division of Pathology, Foundation IRCCS (Scientific Institute \\ for Research Hospitalization and Health Care), Ca' Granda General Hospital, University of Milan, Milan, Italy
}

Received May 14, 2012; Accepted August 16, 2012

DOI: $10.3892 / \mathrm{ol} .2012 .880$

\begin{abstract}
The majority of stage I lung cancer patients undergo a complete resection of their tumor; however, they still harbor a considerable risk of mortality due to recurrences. A correlation between the presence of lymph node micrometastases and poor prognosis has been observed. The aim of this study was to correlate the lymph node molecular staging with the 5-year survival and disease-free interval following pulmonary lobectomy for non-small cell lung cancer (NSCLC). A quantitative real-time reverse transcriptase-polymerase chain reaction (RT-PCR) for carcinoembryonic antigen (CEA) mRNA was performed on primary lung tumors and regional lymph nodes from 55 surgically resected NSCLC patients classified as clinical stage I. CEA mRNA was found to be present in all the primary tumors. RT-PCR revealed the presence of cancer cells in the lymph nodes of 20 patients $(36.3 \%)$ and routine staining detected lymph node metastases in 11 patients. Significant differences in survival and disease-free intervals were observed in patients with lymph node micrometastases versus patients with negative lymph nodes $(\mathrm{P}=0.0026$ and $\mathrm{P}=0.0044$, respectively). Multivariate analyses confirmed that micrometastases were an independent predictor for worse prognosis $(\mathrm{P}=0.0098)$ and a short disease-free interval $(\mathrm{P}=0.0137)$. This study demonstrated strong correlations between the molecular detection of lymph node micrometastases and 5-year survival rates and disease-free interval in patients who underwent pulmonary lobectomy for early-stage lung cancer.
\end{abstract}

Correspondence to: Dr Paolo Mendogni, Thoracic Surgery and Lung Transplantation Unit, Foundation IRCCS (Scientific Institute for Research Hospitalization and Health Care), Ca' Granda General Hospital, University of Milan, 35 Via Francesco Sforza, Milan I-20122, Italy

E-mail: paolo.mendogni@unimi.it

Key words: carcinoembryonic antigen, lung cancer, lymph nodes, polymerase chain reaction, thoracic surgery

\section{Introduction}

Despite progress in early detection, radical surgical treatments and multimodal therapeutic approaches, the prognosis of lung cancer remains poor. The prognosis of patients with non-small cell lung cancer (NSCLC) is determined by the disease stage as defined by the International Association for the Study of Lung Cancer. However, this classification is not a precise prediction tool compared to those used for other primary cancers. For example, patients with completely excised stage I NSCLC develop local relapses in up to $30 \%$ of cases and have a 5-year survival rate of $58-73 \%$ (1). This distinctive NSCLC behavior has encouraged the assessment of a number of pathological and laboratory markers which may offer supplementary prognostic information, including epidermal growth factor receptors, angiogenic growth factors, the K-ras oncogene and disseminated tumor cells. Certain studies have suggested that micrometastases, which have not yet been detected by conventional lymph node histopathological examination, may offer additional prognostic information (2-4). A number of molecular methods, including the polymerase chain reaction (PCR) for p53 and K-ras and the reverse transcriptase-polymerase chain reaction (RT-PCR) for carcinoembryonic antigen (CEA), cytokeratin 7 (CK7), cytokeratin 19 (CK19) and mucin 1 (MUC1) have been used for the detection of micrometastases in lymph nodes. The prognostic role of these markers remains controversial and there is no agreement as to whether molecular technologies should be used during routine pathological evaluation (5).

The purpose of this prospective study was to determine the impact of lymph node micrometastases, detected with quantitative real-time RT-PCR assays for CEA mRNA, on 5 -year survival in patients undergoing complete resection of clinical stage I NSCLC. This study follows a preliminary study published in 2005 which focused on the disease-free interval (6).

\section{Materials and methods}

Patients. Mediastinal and hilar lymph nodes from clinical stage I NSCLC patients treated with pulmonary lobectomy 
at the Unit of Thoracic Surgery and Lung Transplantation (Ca' Granda General Hospital, Milan, Italy) between October 2000 and February 2004 were studied following Ethics Committee approval. All patients had been staged clinically by means of chest, abdominal and brain computed tomographic scans, positron emission tomography scans, bronchoscopy and mediastinoscopy (if mediastinal lymph node enlargement or positivity for PET were detected). Patients with a history of previous malignancy or a forced expiratory volume in $1 \mathrm{sec}$ $\left(\mathrm{FEV}_{1}\right)<80 \%$ of the predicted value, were excluded. Consent was obtained from each patient. The patient population included a cluster of NSCLC cases previously reported and a set of additional patients that had been studied prospectively following the original publication (3).

Study protocol. The study protocol was extensively reported in our previous study (6) and is summarized as follows. Systematic lymphadenectomy was performed immediately after thoracic incision and was followed by pulmonary lobectomy. Each lymph node was divided into two sections; one section was frozen at $-80^{\circ} \mathrm{C}$ and the second section was formalin-fixed. A fragment of the primary tumor was frozen. The standard curve was constructed from a mix of lymphocytes from a healthy donor and CEA-expressing MCF-7 cells (American Type Collection, Rockville, MD, USA). Specimens from cell lines, control lymph nodes, lymph nodes from cancer patients and primary tumors were homogenized (TRIzol solution; Invitrogen, Carlsbad, CA, USA) and spectrophotometry determined the purity and quantity of the mRNA extracted. Total mRNA was reverse-transcribed in DNA with reagents from Applied Biosystems (Foster City, CA, USA). The ABI Prism 7700 Sequence Detection System (Applied Biosystems) was used for the quantitative assessment of CEA. During the DNA polymerization, the TaqMan (Applied Biosystems) probe was hydrolyzed and fluorescence was emitted. The charge-coupled device camera on the Prism 7700 device continuously collected the fluorescence emissions during the amplification cycles. The threshold cycle $(\mathrm{Ct})$ was noted when the fluorescence signal reached 10 times the standard deviation of the background. The linear regression of the standard curve determined the number of CEA-positive cells. All PCRs were performed in duplicate (Universal TaqMan 2X PCR Mastermix; Applied Biosystems) in a volume containing $0.1 \mu \mathrm{mol} / 1$ of TaqMan probe and $0.3 \mu \mathrm{mol} / 1$ of each primer. The thermal profile included $2 \mathrm{~min}$ at $50^{\circ} \mathrm{C}, 10 \mathrm{~min}$ at $95^{\circ} \mathrm{C}$ followed by 40 cycles ( 50 for lymph nodes and standard curve points) at $95^{\circ} \mathrm{C}$ for $15 \mathrm{sec}$ and $1 \mathrm{~min}$ at $60^{\circ} \mathrm{C}$. The following sequences of primers were used: forward 5'-ATT CCA TAG TCA AGA GCA TCA CA-3', reverse 5'-GCA AAT GCT TTA AGG AAG AAG-3' and TaqMan probe 5'-(6-FAM) TGA AAT GAA GAA ACT ACA CCA GGG CTG CTA TAT (TAMRA)-3' for CEA (National Center for Biotechnology Information accession number NM_004363) and forward 5'-TCC TTC CTG GGC ATG GAG-3', reverse 5'-AGG AGG AGC AAT GAT CTT GAT CTT-3' and TaqMan probe 5'-(6-FAM) CCT GTG GCA TCC ACG AAA CTA CCT TC-(TAMRA)-3' for ACTB (National Center for Biotechnology Information accession number NM_001101). Threshold cycle values $<37.42$ were considered significant for the presence of cancer cells in the lymph nodes. The formalin-fixed specimens underwent routine hematoxylin
Table I. Patient characteristics.

\begin{tabular}{lc}
\hline Variable & Data \\
\hline No. of patients & 55 \\
Male:female ratio & $42: 13$ \\
Average age (range), years & $65.1(36-80)$ \\
Tumor histotype & \\
Adenocarcinomas & 32 \\
Squamous cell carcinoma & 17 \\
Large cell carcimoma & 4 \\
Adenosquamous carcinoma & 2 \\
Median follow-up, months & 45.8 \\
Recurrences & 20 \\
Cancer-related mortalities & 15 \\
\hline
\end{tabular}

and eosin (H\&E) staining. Following surgery, the patients were examined every 2 months in the first year and every 3 months thereafter. If recurrence was suspected, the appropriate investigations were undertaken. Adjuvant chemotherapy and/or radiotherapy were administered following confirmation of recurrence. The survival rate and disease-free interval were recorded. The follow-up was censored in the case of a second malignancy. All the patients were reclassified according to the seventh edition of the TNM classification for lung cancer (1).

Statistical analysis. Survival functions were estimated using the Kaplan-Meier method and the differences were evaluated by the log-rank or the Wilcoxon test, as appropriate. The Cox proportional hazards test was used to identify the factors that influenced the survival rate and disease-free interval. The regression analysis was applied to standard curves and the analysis of variance was used where indicated.

\section{Results}

Three-hundred and thirty-one consecutive NSCLC stage I patients were evaluated. Sixty-one patients with previous malignancy, 95 with $\mathrm{FEV}_{1}<80 \%, 24$ requiring pneumonectomy and 13 patients requiring sleeve-lobectomy were excluded and 27 patients did not provide consent. A set of 15 patients with a final diagnosis of benign disease, 22 patients with a diagnosis of pulmonary carcinoid tumor and 2 diagnosed with small-cell lung cancer were also excluded. Four patients had specimens not adequately frozen, 4 patients were upstaged to T4 and 9 patients were found to have unexpected N2 disease at routine staining, causing their removal from the trial. The remaining 55 patients constituted the study group.

The 55 patients included 42 males and 13 females. The mean age was 65.1 years (range, $36-80$ years). The NSCLC tumors included $32(58.1 \%)$ adenocarcinomas, 17 (30.9\%) squamous cell carcinomas, 4 large cell carcinomas (7.3\%) and $2(3.7 \%)$ adenosquamous carcinomas. Patients' characteristics are shown in Table I.

A total of 609 lymph nodes were examined with H\&E staining and quantitative real-time RT-PCR for CEA mRNA. 
Table II. N factor according to hematoxylin and eosin staining and quantitative real-time RT-PCR for CEA mRNA.

\begin{tabular}{lcr}
\hline pN factor & Hematoxylin and eosin staining & Real-time RT-PCR for CEA mRNA \\
\hline N0 & 44 & 35 \\
N1 & 11 & 5 \\
N2 & 0 & 15 \\
\hline
\end{tabular}

Data are presented as number of patients. RT-PCR, reverse transcriptase-polymerase chain reaction; CEA, carcinoembryonic antigen.

Table III. Survival and disease-free interval multivariate analysis.

\begin{tabular}{lccc}
\hline Absence of micrometastases & RR & Standard error & 95\% CI \\
\hline Survival & -1.021 & 0.416 & -1.916 to -0.242 \\
Disease-free interval & -0.804 & 0.339 & -1.526 to -0.163 \\
\hline
\end{tabular}

RR, relative risk; CI, confidence interval.

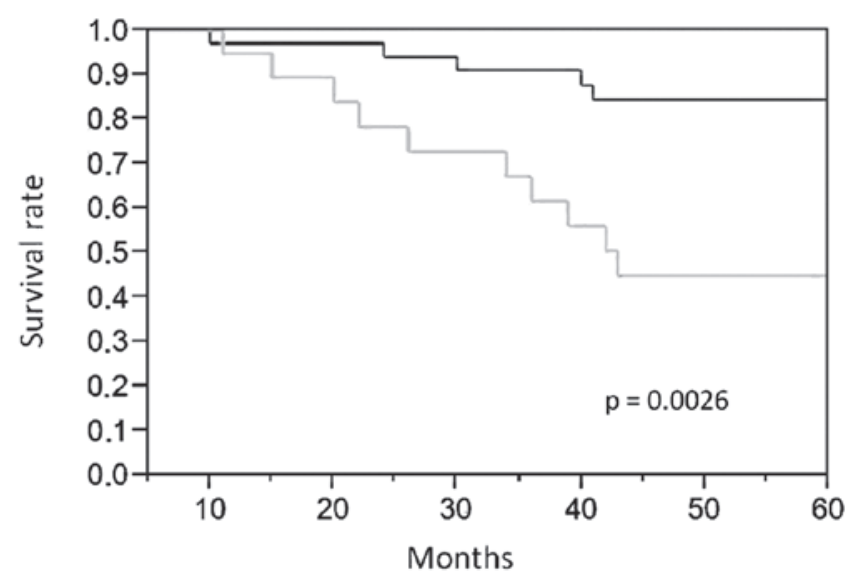

Figure 1. Kaplan-Meier plot. Survival curves revealing significant survival differences $(\mathrm{P}=0.0026)$ between patients with intra-thoracic lymph node micrometastases (gray) and patients without micrometastases (black).

The lymph nodes from the 55 patients were pathologically staged by routine histopathological examination as $\mathrm{pN} 0=44$ and $\mathrm{pN} 1=11$. RT-PCR for CEA mRNA revealed the presence of cancer cells in 20 patients $(36.3 \%)$. Table II details the $\mathrm{N}$ status according to morphologic and molecular procedures. CEA transcript levels were detected in all the primary tumor specimens, with values ranging from 0.044 to 1782.88 .

Contingency analyses did not reveal any correlation between the presence of micrometastases and the tumor mass characteristics (pathological stage T1 or T2), histotype, tumor grade, age, gender and levels of CEA mRNA in the primary cancer.

The mean follow-up time of the 55 enrolled patients was 45.8 months. There were no patients lost to follow-up, cancer recurrence occurred in 20 patients, and 15 patients succumbed to their cancer. Statistically significant survival differences were observed between patients with and without

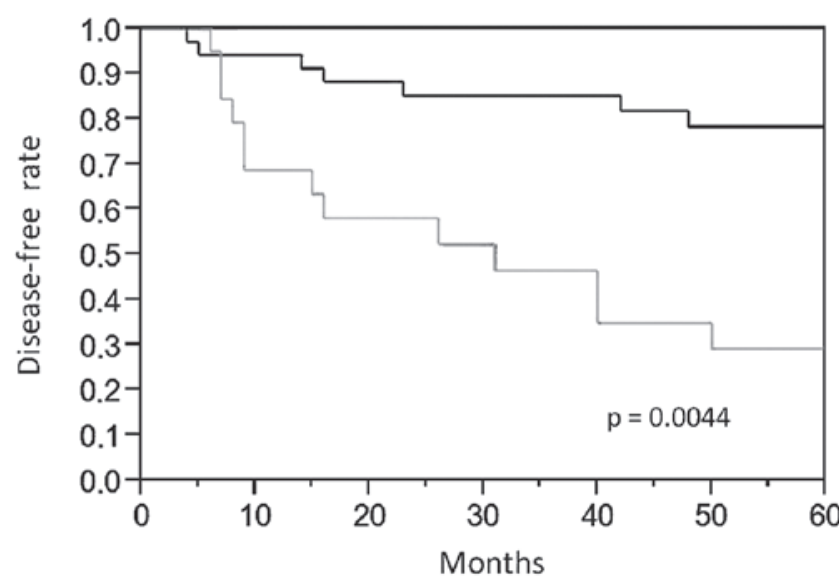

Figure 2. Kaplan-Meier plot. Disease-free interval curves demonstrating significantly worst prognosis $(\mathrm{P}=0.0044)$ in patients with micrometastases (gray) versus patients without micrometastases in the intra-thoracic lymph nodes (black).

lymph node micrometastases $(\mathrm{P}=0.0026$; Fig. 1$)$. There were also statistically significant differences in the disease-free intervals between patients with and without micrometastases $(\mathrm{P}=0.0044$; Fig. 2). Cox regression multivariate analyses of age, gender, $\mathrm{T}$ status, histology and grading revealed that the presence of micrometastases was an independent predictor for worse prognosis $(\mathrm{P}=0.0098)$ and shorter disease-free interval $(\mathrm{P}=0.0137$; Table III).

\section{Discussion}

Lymph node metastases are indicative of a poor prognosis in NSCLC, as well as in other solid tumors, as they provide clear evidence of systemic dissemination, a factor that currently influences the prognosis and therapy. The standard procedure to evaluate lymph node metastases from solid tumors is histopathological analysis, although a more accurate process 
is advisable. In 1948, Saphir and Amromin reported that a limited number of sections taken from axillary lymph nodes following breast cancer surgery, were not sufficient enough to establish whether metastases were present or not (7). The study reviewed lymph nodes which demonstrated no tumors in standard examinations through serial sectioning, hence the metastases that were revealed were named 'obscure' axillary lymph node metastases. Since then, the presence of tumor cells in axillary lymph nodes, which were initially assessed as negative on standard examinations, have been reported; however, their prognostic significance remains unclear. The origin of such indeterminate significance may be related to the distinctive behavior of the isolated tumor cells (or micrometastases), which remains to be investigated. Animal experiments have demonstrated that micrometastases are suppressed by apoptosis and that primary tumor removal induces angiogenesis, leading to metastatic development (8). Research on colorectal cancer has revealed that, in the absence of the primary tumor, both vascular density and metabolic activity in the metastases are increased, while the level of apoptosis is decreased (9).

Several studies have evaluated the presence of micrometastases within intra-thoracic lymph nodes by immunohistochemistry using various antibodies. A recent study reviewed 13 trials correlating micrometastases and survival, in addition to the authors' personal experience (10). This meta-analysis of 835 NSCLC cases revealed no significant correlation between lymph node micrometastases and survival. The result is not surprising in our opinion: the analyzed studies utilized a mosaic of different antibodies, each with specific and different points of strength and weakness. A major problem is the use of immunohistochemistry; such a procedure requires a meticulous and expert pathologist reading thousands of slides, therefore personal interpretation is often a problem. This is the same problem that we encountered in a trial correlating bone marrow micrometastases and survival, the results of which were negative (11).

Efforts have been made to detect lymph node micrometastases at molecular levels and quantitative real-time RT-PCR has been shown to be more sensitive than immunohistochemistry for the detection of micrometastases in patients with solid tumors $(12,13)$. In 2002, D'Cunha et al revealed the effectiveness of quantitative real-time RT-PCR using CEA mRNA in detecting tumor cells in lymph nodes of patients with NSCLC (14). Maeda et al, following the analysis of several molecular markers, evaluated the sensitivity of CEA mRNA for nodal metastases (14). The study, published in 2006, revealed that the slices without tumor cells and lymph nodes obtained from thymoma patients showed no amplification. By contrast, CEA mRNA was detected by RT-PCR in all primary NSCLC and positive lymph nodes as well as in $25 \%$ of the H\&E-negative lymph nodes (15). This study supports our previous paper published in 2005. Following the determination of a threshold cycle in the 14 control lymph nodes, we also detected CEA mRNA in all the primary NSCLC samples, all the positive lymph nodes and in $36 \%$ of patients with negative lymph nodes in routine staining (6). Our preliminary study reported a positive correlation between early cancer recurrence and CEA mRNA detection in the intra-thoracic lymph nodes $(\mathrm{P}=0.021)$. The present study contained an addi- tional set of patients who completed the 5-year follow-up. The results were encouraging and congruent with the preliminary report. Survival rates, as well as the disease-free intervals, were clearly affected by CEA mRNA detection in the intrathoracic lymph nodes. Moreover, the multivariate analysis identified the micrometastases to be a negative predictor for survival and disease-free interval. Such results may have an essential clinical relevance, for example, molecular diagnosis of node involvement may be useful for selecting appropriate candidates from among $\mathrm{pN} 0-1$ patients to receive postoperative chemotherapy.

The present study has several points of strength. The marker CEA is effective in the identification of NSCLC cells in the intra-thoracic lymph nodes and false-negative results did not occur in our results but were observed in other studies. The RT-PCR technology is relatively simple and above all an automated procedure, avoiding any problems relating to personal interpretation. The clinical controls were also meticulous, no patients were lost to the follow-up and survival was unaffected by poor respiratory function.

The weakest point of this study was the number of patients, which prevented any significant comparisons between TNM and molecular staging. A large multicentre trial should further investigate these findings, considering that the data from the surviving patients were encouraging.

In our opinion, the molecular intra-thoracic lymph node staging is a promising new frontier in NSCLC classification which may identify patients requiring specific treatment. In this setting, the development of the loop-mediated isothermal amplification method (which was recently introduced as a fast diagnostic procedure for infectious disorders) may prove significant as it has been successfully tested for detecting CEA mRNA in intra-thoracic lymph nodes. Such new rapid procedures may be proposed during mediastinoscopy or transbronchial preoperative lymph node staging by selecting patients who require neoadjuvant therapy (16).

In conclusion, the present prospective study provided evidence for a strong correlation between the molecular detection of intra-thoracic lymph node micrometastases and 5-year cancer specific survival as well as the disease-free interval in patients who underwent pulmonary lobectomy for early-stage NSCLC.

\section{Acknowledgements}

The authors wish to acknowledge Professor Maria Martellini, Mr. Francesco Caridei and Mrs. Maria Grazia Vitali for their support.

\section{References}

1. Rami-Porta R, Crowley JJ and Goldstraw P: The revised TNM staging system for lung cancer. Ann Thorac Cardiovasc Surg 15: 4-9, 2009.

2. Salerno CT, Frizelle S, Niehans GA, HO SB, Jakkula M, Kratzke RA and Maddaus MA: Detection of occult micrometastases in non-small cell lung carcinoma by reverse transcriptase-polymerase chain reaction. Chest 113: 1526-1532, 1998.

3. Bonavina L, Soligo D, Quirici N, Bossolasco P, Cesana B, Lambertenghi Deliliers $G$ and Peracchia A: Bone marrowdisseminated tumor cells in patients with carcinoma of the esophagus or cardia. Surgery 129: 15-22, 2001. 
4. Ruffato A, Mattioli S, Pileri S, Daddi N, D'Ovidio F, Pilotti V and Tazzari P: Do bone marrow isolated tumor cells influence long-term survival of non-small cell lung cancer? Eur J Cardiothorac Surg 35: 463-468, 2009.

5. Marchevsky AM, Qiao JH, Krajisnik S, Mirocha JM and McKenna RJ: The prognostic significance of intranodal isolated tumor cells and micrometastases in patients with non-small cell carcinoma of the lung. J Thorac Cardiovasc Surg 126: 551-557, 2003.

6. Nosotti M, Falleni M, Palleschi A, Pellegrini C, Alessi F, Bosari S and Santambrogio L: Quantitative real-time polymerase chain reaction detection of lymph node lung cancer micrometastasis using carcinoembryonic antigen marker. Chest 128: 1539-1544, 2005.

7. Saphir O and Amromin GD: Obscure axillary lymph-node metastasis in carcinoma of the breast. Cancer 1: 238-241, 1948.

8. O'Reilly MS, Holmgren L, Chen C and Folkman J: Angiostatin induces and sustains dormancy of human primary tumors in mice. Nat Med 2: 689-692, 1996.

9. Peeters CF, de Waal RM, Wobbes T and Ruers TJ: Metastatic dormancy imposed by the primary tumor: does it exist in humans? Ann Surg Oncol 15: 3308-3315, 2008.

10. Marchevsky AM, Gupta R, Kusuanco D, Mirocha J and McKenna RJ Jr: The presence of isolated tumor cells and micrometastases in the intrathoracic lymph nodes of patients with lung cancer is not associated with decreased survival. Hum Pathol 41: 1536-1543, 2010.

11. Nosotti M, Tosi D, Palleschi A, Rosso L, Mendogni P and Santambrogio L: Immunocy tochemical detection of occult tumor cells in the bone marrow: prognostic impact on early stages of lung cancer. Eur Surg Res 41: 267-271, 2008.
12. Noguchi S, Aihara T, Nakamori S, Motomura K, Inaji H, Imaoka $\mathrm{S}$ and Koyama $\mathrm{H}$ : The detection of breast carcinoma micrometastases in axillary lymph nodes by means of reverse transcriptase-polymerase chain reaction. Cancer 74: 1595-1600, 1994.

13. Kubota K, Nakanishi H, Hiki N, et al: Quantitative detection of micrometastases in the lymph nodes of gastric cancer patients with real-time RT-PCR: a comparative study with immunohistochemistry. Int J Cancer 105: 136-143, 2003.

14. D'Cunha J, Corfits AL, Herndon JE II, et al: Molecular staging of lung cancer: real-time polymerase chain reaction estimation of lymph node micrometastatic tumor cell burden in stage I non-small cell lung cancer - preliminary results of Cancer and Leukemia Group B Trial 9761. J Thorac Cardiovasc Surg 123: 484-491, 2002.

15. Maeda J, Inoue M, Okumura M, et al: Detection of occult tumor cells in lymph nodes from non-small cell lung cancer patients using reverse transcription-polymerase chain reaction for carcinoembryonic antigen mRNA with the evaluation of its sensitivity. Lung Cancer 52: 235-240, 2006.

16. Maeda J, Inoue M, Nakabayashi K, et al: Rapid diagnosis of lymph node metastasis in lung cancer with loop-mediated isothermal amplification assay using carcinoembryonic antigenmRNA. Lung Cancer 65: 324-327, 2009. 